OPEN ACCESS

Edited by:

Eva M. Top,

University of Idaho, United States

Reviewed by:

Ruichao Li,

Yangzhou University, China

Francisco Dionisio,

University of Lisbon, Portugal

Thibault Stalder,

University of Limoges, France

*Correspondence:

Tal Dagan

tdagan@ifam.uni-kiel.de

Specialty section:

This article was submitted to

Evolutionary and Genomic

Microbiology,

a section of the journal

Frontiers in Microbiology

Received: 03 May 2020

Accepted: 05 August 2020

Published: 04 September 2020

Citation:

Hülter NF, Wein T, Effe J,

Garoña A and Dagan T (2020) Intracellular Competitions Reveal

Determinants of Plasmid Evolutionary

Success. Front. Microbiol. 11:2062.

doi: 10.3389/fmicb.2020.02062

\section{Intracellular Competitions Reveal Determinants of Plasmid Evolutionary Success}

\author{
Nils F. Hülter, Tanita Wein, Johannes Effe, Ana Garoña and Tal Dagan* \\ Institute of General Microbiology, Kiel University, Kiel, Germany
}

Plasmids are autonomously replicating genetic elements that are ubiquitous in all taxa and habitats where they constitute an integral part of microbial genomes. The stable inheritance of plasmids depends on their segregation during cell division and their longterm persistence in a host population is thought to largely depend on their impact on the host fitness. Nonetheless, many plasmids found in nature are lacking a clear trait that is advantageous to their host; the determinants of plasmid evolutionary success in the absence of plasmid benefit to the host remain understudied. Here we show that stable plasmid inheritance is an important determinant of plasmid evolutionary success. Borrowing terminology from evolutionary biology of cellular living forms, we hypothesize that Darwinian fitness is key for the plasmid evolutionary success. Performing intracellular plasmid competitions between non-mobile plasmids enables us to compare the evolutionary success of plasmid genotypes within the host, i.e., the plasmid fitness. Intracellular head-to-head competitions between stable and unstable variants of the same model plasmid revealed that the stable plasmid variant has a higher fitness in comparison to the unstable plasmid. Preemptive plasmid competitions reveal that plasmid fitness may depend on the order of plasmid arrival in the host. Competitions between plasmids characterized by similar stability of inheritance reveal plasmid fitness differences depending on the plasmid-encoded trait. Our results further reveal that competing plasmids can be maintained in coexistence following plasmid fusions that maintain unstable plasmid variants over time. Plasmids are not only useful accessory genetic elements to their host but they are also evolving and replicating entities, similarly to cellular living forms. There is a clear link between plasmid genetics and plasmid evolutionary success - hence plasmids are evolving entities whose fitness is quantifiable.

Keywords: genome evolution, lateral gene transfer, antibiotics resistance, extrachromosomal DNA, homologous recombination

\section{INTRODUCTION}

Extrachromosomal genetic elements are ubiquitous in prokaryotes and eukaryotes. Viruses, mitochondria, bacteriophages and plasmids are examples for extrachromosomal genetic elements that depend on a hosting cell for reproduction where they replicate independently of the chromosome. Some elements are beneficial for their host (e.g., eukaryotic organelles), others are 
characterized as parasitic (e.g., viruses) or selfish (e.g., transposons). It is commonly agreed that extrachromosomal elements evolve by 'descent with modification' where genetic modifications are heritable over time and generations. Nonetheless, studying the effects of natural selection on the evolution of extrachromosomal elements is a challenging concept; how to disentangle the evolutionary success of extrachromosomal elements from that of their host remains an open question in evolutionary biology.

Here we focus on prokaryotic plasmids, which have been extensively studied due to their contribution to gene transfer during microbial evolution. Plasmids encoding for resistance mechanisms to antibiotics confer an immediate advantage to their host fitness under selection for antibiotics resistance. Such plasmids are often found in ecosystems exposed to fluctuating levels of antibiotics (e.g., Pham et al., 2018; González-Plaza et al., 2019). Nonetheless, many plasmids found in nature are lacking a clear trait that is advantageous to the host (i.e., 'cryptic' plasmids). For example, a recent study of the rat cecum metamobilome revealed hundreds of small novel cryptic plasmids (Jørgensen et al., 2014). Such small cryptic plasmids may carry very few genes next to their replication machinery (e.g., Carneiro et al., 2016; Zhou et al., 2018). Plasmid persistence (i.e., the plasmid presence within a microbial population over time) in the absence of positive selection is mainly dependent on stable plasmid replication and segregation, that is, reproduction and vertical inheritance (Bergstrom et al., 2000; Paulsson, 2002). An important property of plasmids is the number of plasmid copies in the cell (plasmid copy number; PCN) that is tightly regulated by copy number control mechanisms encoded by the plasmid (e.g., Pinkney et al., 1988; Kittell and Helinski, 1991; Wagner et al., 1992). Plasmid replicons are thus typically polyploid hence the plasmid population within a host cell may comprise multiple plasmid variants (i.e., alleles) due to independent emergence of mutations in the plasmid copies (Bedhomme et al., 2017; Rodriguez-Beltran et al., 2018). The PCN can be variable among cells in both space and time and it has important implications for the segregation of plasmid variants (Ilhan et al., 2019). Plasmid segregation into daughter cells during cell division may be facilitated by active partition mechanisms (Baxter and Funnell, 2014) or plasmid diffusion in the cell (Wang, 2017).

Plasmids whose inheritance is unstable may persist in the population, e.g., via infective transmission to plasmid-free cells (Heuer et al., 2007; Hall et al., 2016). Additionally, plasmids may carry functions that confer a competitive advantage to their host, and thus ensure that plasmid-carrying cells will out-compete plasmid-free cells in the population. This includes functions that are beneficial for the host depending on the environmental conditions [e.g., Lactococcus lactis (McKay et al., 1976)], functions that are indispensible for the host regardless of the environmental conditions [e.g., symbiotic lifestyle maintenance (Grote et al., 2016)], and mechanisms for post-segregational killing of plasmid-free cells (Gerdes et al., 1986a,b), which may also lead to the exclusion of competing plasmids (Cooper and Heinemann, 2000). Other factors that may influence plasmid persistence in the population range from positive or negative interactions with the host cell, e.g., with the host replisome
(Sota et al., 2010) or defense mechanisms (e.g., Mahendra et al., 2020), to the transcriptional load of plasmid genes (e.g., Buckner et al., 2018).

Previous studies on the evolution of plasmid persistence in naïve hosts focused mainly on plasmids with a large genome size (e.g., $>50 \mathrm{~Kb}$ ) that encode multiple functions that support the stable plasmid inheritance or the plasmid persistence in the population (e.g., Yano et al., 2016; Bottery et al., 2017; LoftieEaton et al., 2017). In contrast, small plasmids that do not confer a clear function to their host remain largely understudied. Plasmids that have a negligible effect on their host fitness may evolve a stable inheritance and thus become permanent in the population and an integral component of the lineage genome (Wein et al., 2019). However, quantifying plasmid evolutionary success requires us to disentangle the plasmid reproductive and segregation success from that of the host.

Borrowing terminology from evolutionary research of cellular living forms, we hypothesize that Darwinian fitness is key for the plasmid evolutionary success. Here we define Darwinian fitness of plasmids as the average contribution of a specific plasmid genotype to the plasmid allele pool in the next generation. The fitness of plasmids thus relies on their reproduction, i.e., replication in the cell, and in addition, on their segregation i.e., inheritance during cell division. We further propose that the concept of plasmid fitness is key for understanding the existence and evolution of plasmids and other extra-chromosomal genetic elements. Notably, natural selection operates on plasmids in two hierarchical levels; one component of plasmid fitness is the host fitness within the population and the second component is the plasmid fitness within the cell. While the first component has been extensively studied, the second component has been so far largely neglected. Here we present a novel approach to compare the fitness of naturally occurring plasmid backbones within the host cell. Our approach includes pairwise in cellulo competitions between selected variants of the same plasmid backbone. We hypothesize that stable plasmid inheritance is an important determinant of plasmid fitness hence a plasmid variant characterized by stable inheritance is expected to outcompete a plasmid variant whose inheritance is unstable.

\section{RESULTS}

\section{Comparison of Plasmid Fitness Independent of the Bacterial Host Cell}

To study the intracellular dynamics of different plasmid variants, we developed an experimental system that enables us to perform pairwise plasmid competition experiments in Escherichia coli K12 MG1655. For the comparison of plasmid fitness between plasmid variants, we performed pairwise plasmid competitions between two variants of the same model plasmid in two settings: in the direct 'head-to-head' competition, pairs of plasmids are competing upon arrival into the same naïve host cell. In the preemptive competition, an invading plasmid is competed against an endemic plasmid (i.e., a plasmid that is already present in the host cell) (Figure 1A). Our system includes two variants of the same plasmid backbone: pCON and pCON-S. 
A

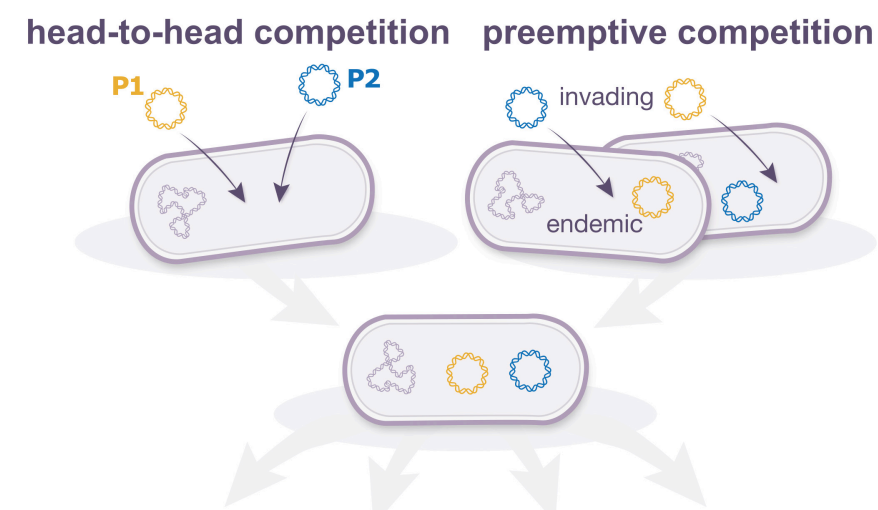

B

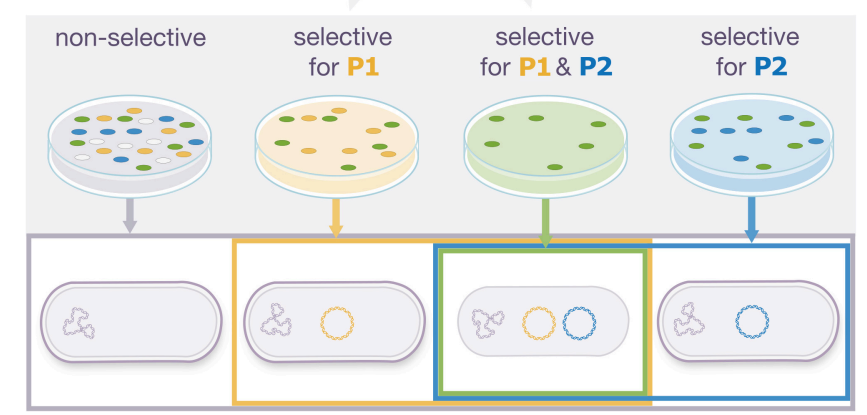

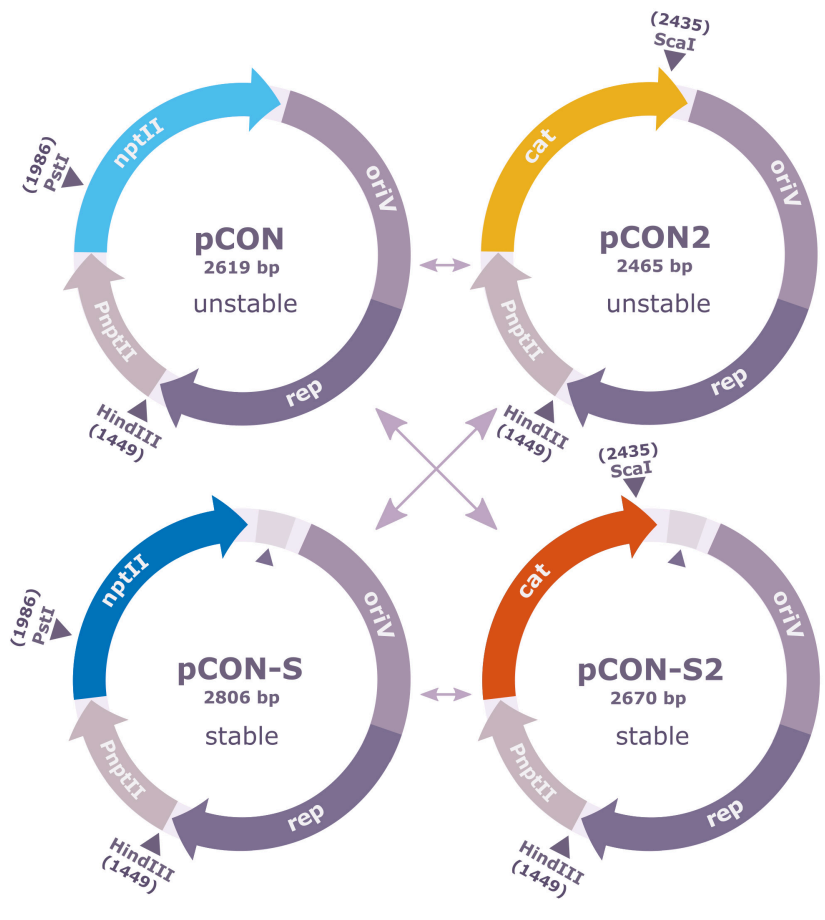

FIGURE 1 | In cellulo comparison of plasmid fitness using pairwise competition experiments. (A) Pairwise competition assay designs. In the head-to-head competition mode, the competing plasmids are introduced into the host simultaneously. In the preemptive competition mode, the competing plasmids are introduced into the host consecutively. Hosts of both plasmids constitute the initial population for the competition. The result of the plasmid competition is evaluated according to the frequency of host types at the end of the experiments. These include plasmid-specific hosts, hosts of both plasmids in coexistence (as well as plasmid-free segregants). (B) Our set of competitor model plasmids comprised two unstable (pCON and pCON2) and two stable plasmids (pCON-S and pCON-2S) derived from the broad-host-range replicon pBBR1 allowing the assessment of plasmid fitness in reciprocal combinations (shown as arrows in the figure). nptll, kanamycin resistance gene; cat, chloramphenicol resistance gene, oriV, origin of replication; rep, replication initiation; Pnptll, native promoter of the nptll gene from Tn5. Relevant restriction sites are indicated. Previously, we showed that pCON instability is caused by transcription-replication conflicts of the resistance gene transcription and the plasmid replication machinery (Wein et al., 2019). The two stable plasmids (pCON-S and pCON-S2) are characterized by the presence of a longer segment between the resistance gene and the origin of replication in comparison to the unstable plasmids (marked by a triangle).

Both plasmids originated from the pBBR1 backbone isolated from Bordetella bronchiseptica (Antoine and Locht, 1992); the pBBR1 plasmid is typically characterized by a small genome size and it is widely spread in diverse environments (Wein et al., 2019). The plasmids pCON and pCON-S are non-mobile, encode the kanamycin resistance gene nptII and have a comparable plasmid copy number ranging between 2 and 6 (Wein et al., 2019, 2020). While pCON is characterized by an unstable inheritance in a population, and accordingly is lost over time, pCON-S is characterized by a stable inheritance in the population over long time scales (Wein et al., 2020). The instability of pCON is a result of transcription-replication conflicts of the plasmid replication machinery and the transcription of the nptII gene (Wein et al., 2019) that is resolved by an insertion between the nptII gene and the oriV region in pCON-S (Wein et al., 2020) (see details in Figure 1B) (Wein et al., 2019, 2020). Both pCON and pCON-S have no measurable effect on host fitness (Wein et al., 2019, 2020). To compete both plasmid variants in the same host, we created pCON2 and pCON-S2 in which we replaced the kanamycin resistance gene nptII with the cat gene that confers resistance to chloramphenicol (Figure 1B). Testing the plasmid stability in an overnight incubation (ca. 8 generations) showed that pCON2 had a loss frequency of $11 \% \pm 3.5(\mathrm{SE} ; n=6)$, which is comparable to pCON, while pCON-S2 had no measurable loss $(n=6)$. Both pCON2 and pCON-S2 had no measurable effect on the host fitness (Supplementary Figure S1). Thus, hosts of all plasmid variants in our experiment had a similar fitness under the tested conditions such that differences in plasmid frequency in the population over time can be attributed to differences in the success of the plasmid to complete a full replication and segregation cycle rather than the fitness of their host.

In the competition experiments, the two plasmid variants were transformed into an E. coli population and the presence of both plasmids was validated according to the host resistance to both antibiotics. Hosts of both plasmids were plated on nonselective media and incubated overnight, which amounts to ca. 25 generations. The frequency of both competing plasmids was estimated from the frequency of hosts harboring the plasmids within the population (i.e., as observed in single colonies). Considering the negligible effect of our model plasmids on the 
host fitness and the minimal number of host cell divisions in the experiment, the observed differences in plasmid frequencies within the population can be interpreted as evidence for differences in the fitness of individual plasmids within the host. Our approach is parallel to the Luria-Delbrück experiment (termed fluctuation test) that was conceived in order to test for a deviation in the frequency of newly emergent chromosomal mutations from the random expectation (Luria and Delbrück, 1943). Using the same logic, if the reproductive success of both competing plasmids is similar then their frequency in the host is expected to be the result of random plasmid segregation only, such that the frequency distribution will be similar between the two plasmids across replicates. Alternatively, if plasmid stability were a determinant of plasmid reproductive success then we would expect the stable plasmid to be significantly more frequent in comparison to the unstable plasmid across replicates.

\section{The Stability of Plasmid Inheritance Is a Determinant of Plasmid Fitness}

To evaluate the impact of plasmid stability on the reproductive success (i.e., fitness) of competing plasmids, we performed direct competitions between the unstable pCON and the stable pCONS2 as well as the reciprocal set of pCON2 and pCON-S. Plasmid frequency in the population was measured using the proxy of the proportion of hosts harboring the two plasmids. The results of the head-to-head competition between pCON and pCONS2 reveal that the frequency of the stable pCON-S2 hosts was higher than that of the unstable pCON hosts (Figure 2A). The stable pCON-S2 was present in most replicate populations (28 out of 35), where the majority of the populations harbored only pCON-S2 hosts hence in these populations pCON went extinct. Overall, pCON was observed in only a few populations (10 out of 35) where pCON hosts typically comprised a minority within the host population. One replicate population stood out as an exception, in which pCON hosts comprised the majority of the population. Additionally, cells hosting a combination of both plasmids in a co-existence were observed in 16 of the populations, where they often comprised the majority of hosts and up to $100 \%$ hosts in two of the replicates. The results of the reciprocal head-to-head competition between pCON2 and pCON-S reveal a similar pattern, where the frequency of the stable plasmid pCON-S hosts was higher than that of the unstable pCON2 hosts (Figure 2D). At the end of the competition, the stable plasmid pCON-S was present in most of the replicates (29 out of 36) with eight replicates comprising $100 \%$ pCON-S hosts. The unstable plasmid pCON2 was observed in 16 of the replicates, albeit with a low proportion of hosts $(<23 \%)$. Hosts harboring both plasmids where observed in 27 populations and their frequency was generally high, approaching $100 \%$ of hosts in six of the populations (Figure 2D). Both head-to-head competitions show that when an unstable plasmid is in direct competition with a stable plasmid it may be lost from the population. Furthermore, our results show that the loss frequency of pCON while in competition with a stable plasmid is much higher in comparison to the pCON loss frequency observed in a pCON host-only population. Nevertheless, our results reveal a high frequency of cells that host both plasmids, i.e., where the unstable and stable plasmids coexist.

The emergence of segregants (i.e., plasmid-free cells) was observed in both head-to-head competition experiments. The plasmid loss frequency in the pCON/pCON-S2 competition was quite moderate, affecting $13(36 \%)$ of the populations (Supplementary Figure S2). In comparison, plasmid loss frequency in the reciprocal pCON2/pCON-S competition was pervasive, affecting $29(80,5 \%)$ of the populations with a median of 50\% segregants per population (Supplementary Figure S2).

Overall, the results of the head-to-head competition reveal a significant deviation from the random expectation as the stable plasmid had a higher frequency in the population in comparison to the unstable plasmid across replicates. Consequently, we conclude that the stable plasmid had a higher fitness in comparison to the unstable plasmid. Our results further show that a competition among plasmids in the cell may lead to the exclusion and extinction of unstable plasmids.

\section{Plasmid Fitness Depends on the Order of Plasmid Arrival in the Host}

The establishment of plasmids in a new host may depend on the presence of other resident plasmids in the host (Kusano et al., 1995; Cooper and Heinemann, 2000). To further test if the effect of plasmid stability on plasmid fitness depends on the plasmid order of arrival, we performed preemptive competition experiments where the competitor plasmid - termed here invading plasmid - is transformed into a host population of the contender plasmid - termed here endemic plasmid. The results of the $\mathrm{pCON} / \mathrm{pCON}-\mathrm{S} 2$ preemptive competitions reveal that the stable plasmid pCON-S2 had a higher frequency in the population in comparison to the unstable plasmid pCON, regardless of order of plasmid arrival, i.e., as the endemic or invading plasmid (Figures 2B,C). When pCON was the endemic plasmid, hosts of pCON-S2 comprised the majority in most replicates while pCON hosts went extinct in most populations. In addition, we observed the emergence of hosts where both plasmids were maintained in coexistence, which comprised ca. $100 \%$ of the population in three of the replicates (Figure 2B). When pCON was the invading plasmid, we observed several replicates that included pCON hosts, with five replicates where pCON outcompeted pCON-S2 (Figure 2C). To perform the reciprocal preemptive competition of pCON-S and pCON2 we transformed populations of endemic pCON-S with an invading pCON2, and the other way around. The experiment with pCON$\mathrm{S}$ as the endemic plasmid yielded no transformant colonies, while having pCON2 as the endemic plasmid yielded only a single colony. The overnight pCON2/pCON-S competitions showed that pCON-S hosts comprised the majority of the population, while pCON2 went extinct in all six replicates. Hosts maintaining both plasmids in coexistence were observed with a low frequency (Supplementary Table S2). We note that the pCON2/pCON-S head-to-head competition was characterized by a high proportion of segregants (Supplementary Figure S2), suggesting that the combination of $\mathrm{pCON} 2$ and $\mathrm{pCON}-\mathrm{S}$ cannot be stably maintained within the population. 


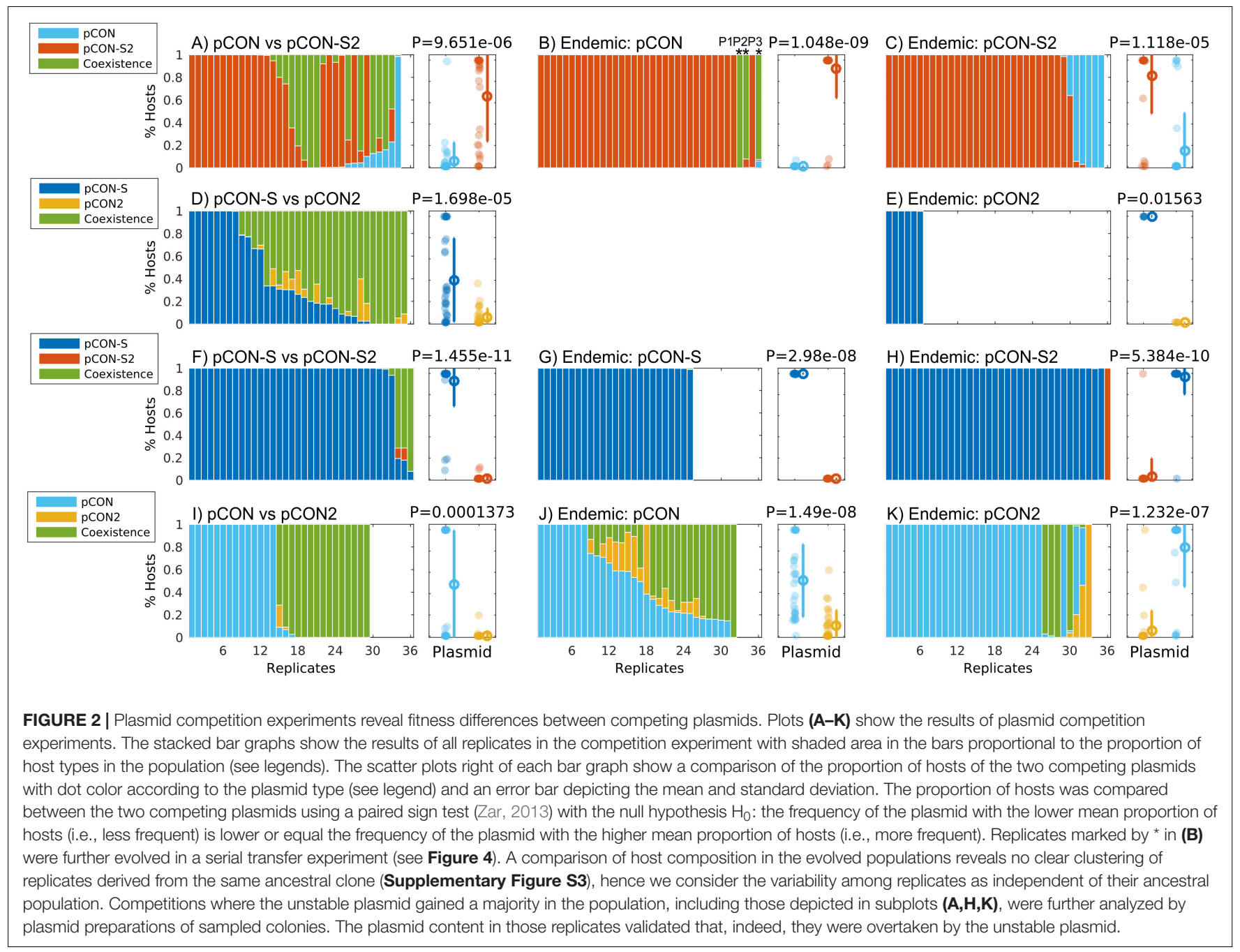

Taken together, the results of preemptive competition experiments demonstrate that the stable plasmid had a higher fitness in comparison to the unstable plasmid, regardless the order of arrival and the plasmid marker genes nptII and cat.

\section{Plasmid Fitness Advantage Depends on a Plasmid Encoded Trait}

Our results so far show that plasmid stability determines plasmid fitness. Nonetheless, plasmids may differ not only with regards to their stability, but also in the traits that they encode. To further examine the effect of plasmidencoded traits on the plasmid fitness, we compared the fitness of plasmids having similar stability but differ in the antibiotic resistance gene that they carry. For that purpose, we performed pairwise competition experiments between the two stable plasmid variants and between the two unstable plasmid variants.

The results of the head-to-head competition between pCON$\mathrm{S}$ and $\mathrm{pCON}-\mathrm{S} 2$ showed that the frequency of pCON-S hosts was higher than that of PCON-S2 hosts, which were observed in only three replicates in a very low frequency (Figure $2 \mathrm{~F}$ and Supplementary Table S2). In addition, we observed the emergence of plasmid coexistence in several replicates with hosts of both plasmids comprising the majority of the population $(>60 \%)$ only in three replicates (Figure 2F). The preemptive competitions performed with both stable plasmids show that pCON-S hosts comprised the majority of replicates regardless of whether pCON-S was the endemic or the invading plasmid (Figures 2G,H). The reciprocal preemptive competition with pCON-S2 as the endemic plasmid resulted in similar dynamics with the majority of replicates (31 out of 36 ) and one exceptional replicate where only pCON-S2 hosts where observed (Figure $\mathbf{2 H}$ and Supplementary Table S2). Plasmid loss in all competition experiments was minimal when pCON-S was the endemic plasmid and slightly higher when pCON-S2 was the endemic plasmid (Supplementary Figure S2).

Comparing the proportion of pCON-S and pCON-S2 hosts in all competition experiments shows that pCON-S maintained a higher frequency in the population regardless of the order of arrival. The results of the competition experiments between stable plasmids thus show that the presence (or invasion) of a 
stable plasmid may lead to the exclusion of an equally stable plasmid type. We observe a clear fitness advantage of the nptIIencoding plasmid over the cat-encoding plasmid despite their equal stability. Whether the expression of $n p t I I$ or cat has an impact on the host fitness under the conditions we applied in our experiments remain unknown. Our results thus indicate that in a competition between equally stable plasmids, other plasmid properties may be important determinants of the plasmid fitness.

The results of the head-to-head competition of both unstable plasmids - pCON and pCON2 - show that half of the replicates included a majority of pCON hosts, while the other half included a majority of host where both plasmids coexisted (Figure 2I). The presence of pCON2 could be observed only in two of the replicates, albeit, at a very small frequency. A similar trend was observed in the results of the preemptive competitions between the two unstable plasmids. When pCON was the endemic plasmid and pCON2 the invading plasmid, the frequency of pCON hosts was higher than the frequency of pCON2 hosts (Figure 2J). The presence of the invading plasmid pCON2 was observed in 19 of the replicates, with a maximum frequency of $60 \%$ (Supplementary Table S2). The presence of pCON and pCON2 was observed in most replicates, with hosts of both plasmids in coexistence making up for the majority in 14 (44\%) of the replicates. In the reciprocal preemptive competition with pCON2 as the endemic plasmid and pCON as the invading plasmid, the frequency of pCON hosts was higher in comparison to the frequency of pCON2 hosts (Figure $2 \mathbf{K}$ ). The presence of pCON2 could be observed only in five replicates with one replicate including only pCON2 hosts. Additionally, we observed the emergence of hosts of both plasmids in coexistence in seven replicates, with four replicates comprising mostly hosts of both plasmids (Figure $2 \mathrm{~K}$ and Supplementary Table S2). The plasmid loss frequency was similar among the three pCON/pCON2 competitions (Supplementary Figure S2). The results of the competition experiments between the two unstable plasmids show that $\mathrm{pCON}$ had a fitness advantage over pCON2. The results of the pairwise competition experiments between the stable or unstable plasmid pairs show that the same plasmid type (i.e., encoding the same trait) had a fitness advantage over the other plasmid type regardless of plasmid stability.

\section{Competing Plasmids Are Maintained via Plasmid Fusion Over Time}

Our results show that different plasmid variants can be maintained in coexistence. Plasmid coexistence may be explained either by plasmid co-residence in the cell or by plasmid recombination (i.e., plasmid fusions). To further investigate the genetic basis of plasmid coexistence, we analyzed the plasmids from double resistant colonies from each of the "head-tohead" competition experiments. In all of the plasmid hosts we observed large plasmids much above the size of our model plasmids (up to $\sim 20 \mathrm{~Kb}$ ). Restriction enzyme digestion analysis showed that these large plasmids corresponded to fusions of both competing plasmids. Hence, the plasmids recombined to form heteromultimeric structures (Figure 3A). We did not observe any apparent re-arrangements or deletions in the plasmid genomes; instead the multimers were composed of the intact plasmids in tandem repeat orientation (Figure 3A). Notably, different heteromultimeric combinations appeared in variable frequencies across plasmid hosts such that no two plasmid populations looked alike. The presence of such diverse combinations of multimers in single hosts suggests that the formation and diversification of plasmid multimers is a dynamic and rapid process. In order to assess whether the observed multimeric plasmids are heritable, we excised a plasmid multimer from an agarose gel and transformed it into an E. coli MG1655 host. Indeed, we obtained a double resistant clone and subsequent DNA analysis validated that the excised plasmid contained a heteromultimeric plasmid fusion. A diversity of plasmid fusions was reconstituted already after overnight incubation, adding further support that the process of plasmid diversification is rapid. Our results thus demonstrate that plasmid coexistence is achieved by plasmid fusions that are heritable.

To test the persistence of such plasmid fusions in the population over time, we performed a short-term evolution experiment of representative populations where hosts of both plasmids comprised the majority of the population. For that purpose, we selected three populations from the $\mathrm{pCON} / \mathrm{pCON}$ S2 competition experiment (Figure 2B). Eight replicates from each population were evolved in serial transfer for 4 days, that is, ca. 80 generations. The experiment results reveal that hosts of both plasmids in coexistence were maintained in the population over time in most replicates (Figure 4). The fixation of plasmid coexistence (i.e., $>90 \%$ of hosts) was observed in two replicates, while in the other replicates we observed the emergence of separate populations of pCON and pCON-S2 hosts. In four of the replicates, hosts of pCONS2 constituted the majority of the population at the end of the experiment. Our results thus indicate that plasmid coexistence is a reversible state where both stable and unstable plasmids are able to segregate from hosts carrying plasmid fusions.

To investigate the genetics of the plasmid coexistence in the evolved populations, we analyzed plasmid DNA of representative hosts from eight of the evolved populations (4.1 and 4.3). Again, we observed heteromeric plasmid fusions in all of the analyzed samples, albeit at a much reduced diversity (Figure 3B). The decreased diversity of plasmid multimers allowed us to resolve some of the plasmid fusions into distinct plasmid compositions. Examples of heteromultimeric structures include oligomers where two pCON plasmid molecules were fused with one molecule of plasmid pCON-S2, forming a heterotrimer (Figure 3B). Notably, we frequently observed faint bands that matched the size of supercoiled monomers of the model plasmids, suggesting that the plasmid coexistence state is either accompanied by the persistence of plasmid monomers or their reappearance through intramolecular recombination within multimers (Supplementary Figure S4B). This observation is further supported by the segregation into plasmid-specific hosts we observed in the serial transfer experiment (Figure 4). Our results thus show that the competing plasmids can be maintained in coexistence following plasmid fusions that can maintain the unstable plasmid over time. Furthermore, we show that plasmid 


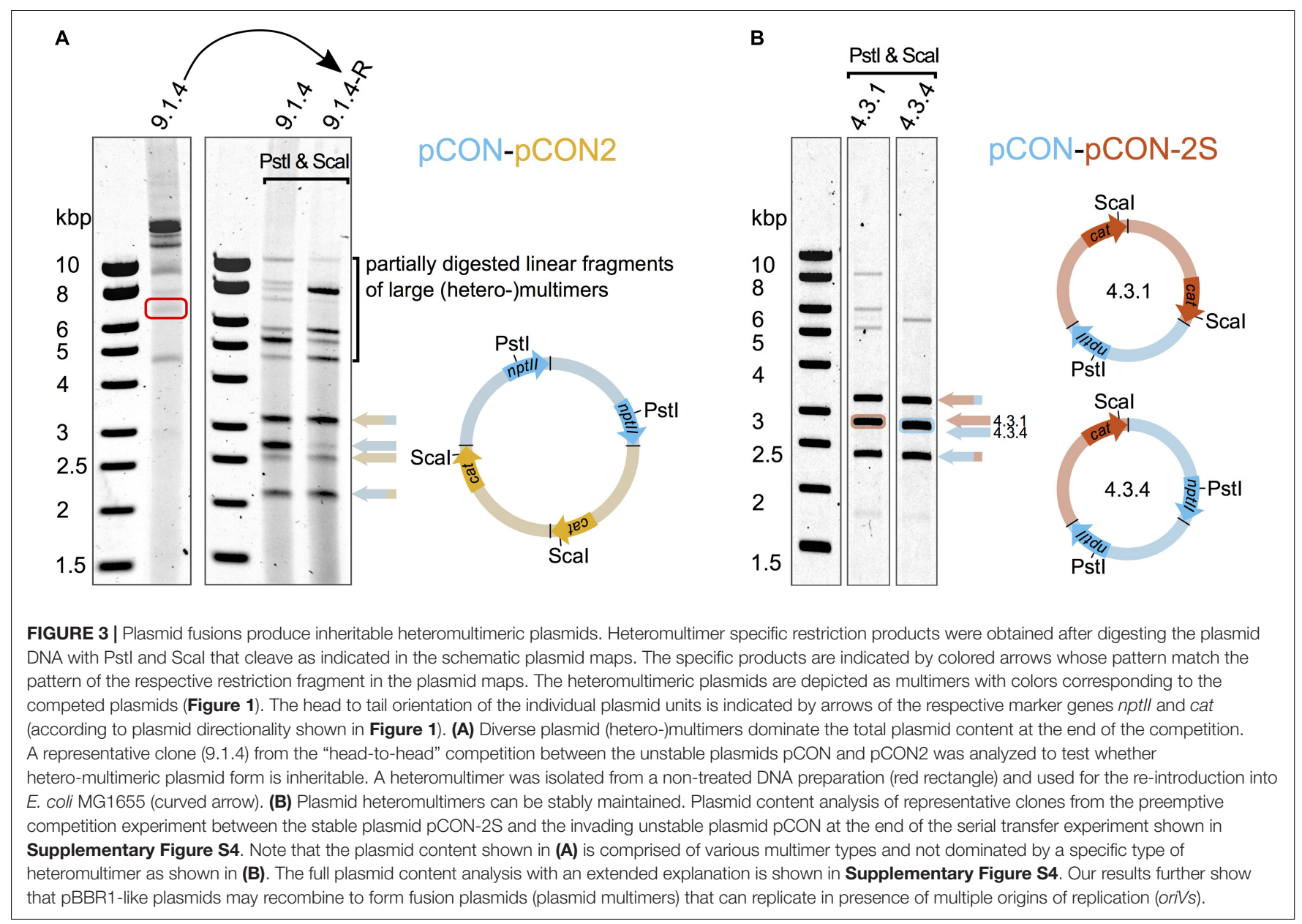

fusion diversity is reduced over time, which indicates that certain plasmid fusions may have a fitness advantage over others.

\section{DISCUSSION}

Stable reproduction and segregation during cell division are essential for plasmid persistence in the population over time. Increasing evidence shows that the persistence of mobile plasmids under strong selection for the plasmid-encoded function is mainly governed by vertical inheritance, while lower selection pressure for the plasmid leads to persistence by mobility in the population (e.g., Lopatkin et al., 2016; Hall et al., 2017; Stevenson et al., 2017). Strong selective conditions for the plasmid presence eliminate non-hosts from the population, thus revealing plasmids that maintain (or adapt) a stable inheritance with the host. When the strength of selection is reduced (or eliminated), plasmids whose segregation is incomplete can persist in the population by alternative mechanisms [e.g., mobility (Hall et al., 2016, 2017; Gumpert et al., 2017; Lopatkin et al., 2017)]. Using our approach for direct in cellulo plasmidcompetitions, we demonstrate the importance of plasmid stability for the evolutionary success (i.e., fitness) of plasmids, even under very short time scales. Nevertheless, our results reveal that unstable plasmid variants may, in several cases, rise to a high frequency in the population (e.g., Figure 2C). Previously we suggested that the effect of selection on plasmid alleles is reduced due to random genetic drift of plasmid alleles during cell division - termed segregational drift (Ilhan et al., 2019). Since the dynamics of plasmid alleles under non-selective conditions largely depends on allele frequency in the population (Ilhan et al., 2019), a stable plasmid may still be lost from the population when its initial frequency in the ancestral cell is low. Our results thus support the notion that segregational drift of plasmid alleles may lead, in some cases, to the fixation of inferior plasmid variants in the population.

Plasmid persistence in a host may depend on an interaction with co-residing plasmids (e.g., San Millan et al., 2009, 2014; Silva et al., 2011; Dionisio et al., 2019; Jordt et al., 2020) or on their recombination and fusion with other plasmids. Recombination routes were shown to facilitate the formation of mosaic plasmids, a phenomenon often observed in different plasmids of the same host lineage (e.g., Chaconas and Norris, 2013; Pesesky et al., 2019). Recombination observed between sites in a single plasmid genome may indicate that plasmid recombinants form routinely when host recombination pathways are encoded in the host genome (e.g., Flores et al., 2000). Indeed, plasmid recombination is dependent on the host 


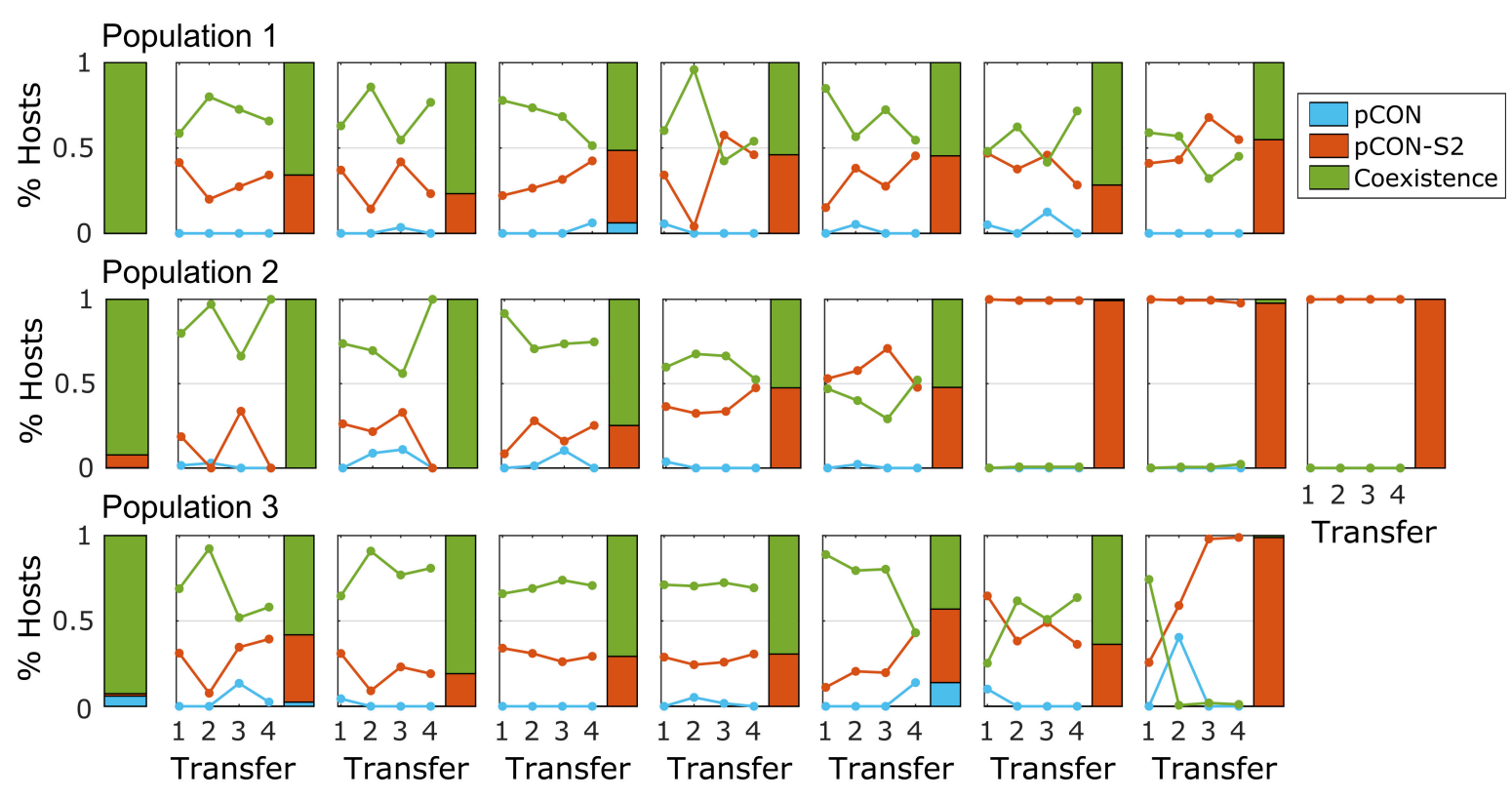

FIGURE 4 | Plasmid coexistence is stable over time. Each subplot shows the results of a short-term evolutionary experiment for a single replicate population with rows corresponding to the three ancestral populations. The solitary stacked bars on the left show the initial host composition in the ancestral population (as in Figure 2). Lines in the plots show the proportion of hosts over the experiment. The host composition at the end of the experiment is shown by a stacked bar. The frequency of segregants (i.e., plasmid loss) reached 100\% in two of the replicates; otherwise, plasmid loss was rather low with a median of $1.4 \%$ segregants per day in the total replicates. The full data set is provided in Supplementary Table S3.

recombination pathways and the formation of plasmid oligomers occurs along the normal lifecycle of plasmids (e.g., James et al., 1982; Chédin et al., 1997). The main cause for the formation of dimers and higher multimers is homologous recombination between spatially linked sister plasmids during or after replication. In contrast, the formation of heteromeric plasmids depends on the recombination of non-sister plasmid molecules (i.e., recombination between two different plasmids). When recombination between non-sister molecules happens early after entry of an invading plasmid (or two plasmids invading at the same time), recombination could lower the effective plasmid copy number of the individual plasmids available for segregation into daughter cells and act as purifying selection toward the existence of the two competing plasmids in a hybrid state (i.e., a heteromer) under double selection. In the competition experiments involving the unstable plasmid pCON2, where we observed higher frequencies of hosts maintaining both plasmids in coexistence (Figures 2D,I,J), such early recombination events might have happened, thereby allowing pCON2 to escape from rapid extinction. Since our experimental design required one step of double selection after plasmid delivery into the host cells, such heteromultimeric forms might have had higher fitness than the individual monomers and were more rapidly purified.

Since the competing plasmid pairs in our experiments share full sequence identity, except for the marker genes nptII and cat, the occurrence of rearrangements within the heteromeric plasmids is expected (Doherty et al., 1983). However, we did not observe any apparent rearrangements in the analyzed heteromultimeric plasmids; all of them comprised units of the model plasmids in tandem orientation. It is likely that the non-identical gene sequences between the plasmid units in a heteromultimer limited recombination to single crossover events, which led to either demultimerization or the formation of higher multimers (i.e., in the recombination between different plasmid molecules). Indeed, the resolution of dimers and higher order multimers back to monomeric forms is possible via intramolecular homologous recombination (Kolodner, 1980) or through site-specific recombination that is mediated by a plasmid - or host - encoded multimer resolution system (e.g., Stark et al., 1992; Fournes et al., 2016). Consequently, the observed stability of heteromultimeric plasmid fusions in our experiments is most likely the result of slow multimer-to-monomer conversion rates by the hostencoded recombination pathways (Summers et al., 1993) and the lack of an apparent multimer resolution site in our model plasmids.

In our experiment, a short-term selective event of both antibiotics was sufficient to select for the larger fusion plasmids. Our results reveal a diversification process of plasmid fusion variants over time that leads to the enrichment of selected variants. Selection for antibiotics resistance may preserve unstable plasmid variants in the population (Wein et al., 2020); our results here show that such unstable plasmids can be maintained in the population over time also under nonselective conditions via plasmid fusion. Plasmid fusion of plasmid alleles conferring resistance to different antibiotics has been invoked as a likely route for the evolution of plasmids carrying 
multiple antibiotics resistance genes (Condit and Levin, 1990). Indeed, the emergence of plasmids conferring resistance to different antibiotics is conceivable, e.g., for plasmids having a high copy number and hence an increased mutational supply (Rodriguez-Beltran et al., 2018), or following gene acquisition by lateral gene transfer [i.e., homologous recombination (Norberg et al., 2011)]. The results of our experiment suggest that once plasmid fusions are formed - in our experiment due to a single selection event - they can enable a stable inheritance of resistance to multiple antibiotics over time also under nonselective conditions. Thus, plasmid stability that is facilitated by plasmid fusion may be a route of plasmid long-term persistence, especially in environments with fluctuating selection pressure.

The comparison of plasmid evolutionary success demonstrates that plasmids variants may differ in their fitness. Our results support the notion that natural selection operates on plasmids in two hierarchical levels: one component is the plasmid fitness within the cell, and the other is the host fitness within the population. Notably, similar principles may apply to the evolution of other extra-chromosomal elements such as mitochondria (Taylor et al., 2002; Gitschlag et al., 2016) and viruses (Krakauer and Komarova, 2003). Our results thus suggest that plasmids in a host cell should be viewed as Darwinian entities whose evolution is governed by basic principles of population genetics, a process partially independent of the hosting bacterial populations. Plasmids are not only useful accessory genetic elements to their host but they are also evolving and replicating entities, similarly to cellular living forms. There is a clear link between plasmid genetics and plasmid evolutionary success - hence plasmids are evolving entities whose fitness can be quantified.

\section{MATERIALS AND METHODS}

\section{Bacterial Strains and Culture Conditions}

The wild-type laboratory E. coli K-12 strain MG1655 was used as the model organism (DSM No. 18039, German Collection of Microorganisms and Cell Cultures, DSMZ). E. coli DH5a (Hanahan, 1983) was used during plasmid constructions and for the analysis of re-introduced plasmids from the plasmid fitness assays (see below). All strains were routinely grown at $37^{\circ} \mathrm{C}$ in lysogeny broth (LB) medium at $250 \mathrm{rpm}$ shaking or on LB-agar plates. Antibiotics for the selection of plasmid carrying host cells were used at the following concentrations: kanamycin $25 \mu \mathrm{g} / \mathrm{ml}$ (pCON and pCON-S hosts), chloramphenicol $10 \mu \mathrm{g} / \mathrm{ml}$ (pCON2 and pCON-S2 hosts), kanamycin $25 \mu \mathrm{g} / \mathrm{ml}$ and chloramphenicol $10 \mu \mathrm{g} / \mathrm{ml}$ (double-plasmid hosts carrying one of the four different possible plasmid combinations).

\section{Plasmid Constructions}

All plasmids in this study were constructed using the Gibson assembly technique (NEBuilder ${ }^{\circledR}$ protocol; New England Biolabs). All primers are listed in Supplementary Table S1. Plasmid pCON-S was derived from plasmid pCON (GenBank accession no. MK697350) by insertion of a $254 \mathrm{bp} \mathrm{PCR} \mathrm{product}$ covering nucleotides 817 to 1044 in pBBR1MCS-5 (GenBank accession no. U25061) into pCON immediately upstream of oriV (Wein et al., 2020). Plasmid pCON2 and pCON2-S were constructed by replacing the open reading frame (ORF) of $n p t I I$ in pCON and pCON-S with the ORF of the chloramphenicol resistance-encoding gene cat, while keeping the original promoter of the nptII gene unaltered. The ORF of the cat gene, corresponding to the nucleotides 1 to 660 of the cat gene ORF from transposon Tn9 (GenBank accession no. V00622.1.), was PCR-amplified from plasmid pPCR-Script Cam SK(+) (Agilent technologies), using the primer pair cat-GA-fw and cat-GA-rv. The nptII-less backbone of pCON-S was PCR-amplified using the primer pair pCONSinvFw and pCONSinvRv. Plasmid pCON2 was constructed by replacing the ORF of the nptII gene in pCON with the ORF of the cat gene (see above). Primers used for the PCR amplification of the cat ORF were cat-pCON2GA-fw and cat-pCON2-GA-rv. The nptII-less backbone of pCON was PCR-amplified using the primer pair pCON2-GA-fw and pCON2-GA-rv.

\section{Plasmid Fitness Competition Experiments}

The success of two plasmid types competed in either the 'headto-head' or preemptive competition mode was determined in single colonies that had grown up non-selectively during $24 \mathrm{~h}$ of growth from single cells that initially carried two plasmids. In order to select for double plasmid carriage and to distinguish between the two plasmids, the competing plasmid pairs harbored different marker genes: nptII in the unstable plasmid pCON and the stable plasmid pCON-S and cat in the unstable plasmid pCON2 and stable plasmid pCON-S2. 'Head-to-head' competitions experiments were initiated by transforming E. coli MG1655 with a mixture of two plasmids at the same time (i.e., pCON and pCON2, pCON and pCON-S2, pCON-S and $\mathrm{pCON} 2$, and $\mathrm{pCON}-\mathrm{S}$ and $\mathrm{pCON}-\mathrm{S} 2$, respectively), giving in total four sets of competition experiments. Likewise, for each preemptive competition experiment E. coli MG1655 already carrying one of our four model plasmids was transformed with a compatible plasmid in terms of plasmid-encoded marker gene, resulting in three sets of plasmid-plasmid combinations that were reciprocally performed (i.e., in total six reciprocal plasmid-plasmid combinations). The four combinations were as follows (endemic versus invading plasmid): pCON versus pCON2, pCON versus pCON-S2, pCON-S versus $\mathrm{pCON} 2$, and pCON-S versus pCON-S2. All plasmids were introduced in E. coli MG1655 by electroporation (1 ng per plasmid DNA per electroporation). To increase the transformation efficiency for double transformations (head-to-head competition experiments) concentrations of 4-10 ng per plasmid DNA were used. Electrocompetent cells were prepared as reported (Dower et al., 1988) and plasmid DNA preparations used for electroporation were isolated from E. coli MG1655 host cells. Colonies resistant to both kanamycin $\left(n p t I I^{+}\right)$and chloramphenicol $\left(\mathrm{cat}^{+}\right)$were selected overnight on selective LB plates. Every competition experiment was initiated with six randomly chosen primary double resistant colonies obtained. The six primary transformants were picked and streaked for single colonies on non-selective LB plates. After 
overnight incubation, for each of the six primary transformants, six single colonies were excised ( $n=36$ replicates per competition experiment). The colonies were resuspended in $1 \mathrm{ml}$ PBS, serially diluted and aliquots plated on non-selective plates [yielding the total cell number per colony $(N)$ ] and plated on kanamycinsupplemented LB plates, chloramphenicol-supplemented plates and plates supplemented with both antibiotics (giving the frequencies of cells being $n p t I I^{+}, \mathrm{cat}^{+}$, and $n p t I I^{+} \mathrm{cat}^{+}$). The proportion of cells carrying only one of the two competing plasmids within one colony were calculated as $P_{p 1}=\left(N_{n p t I I}{ }^{+}\right.$$\left.\mathrm{N}_{\text {nptII }}{ }^{+}{ }_{\text {cat }}{ }^{+}\right) / \mathrm{N}$ for cells carrying either pCON or pCON-S and $P_{p 2}=\left(N_{c a t}{ }^{+}-N_{n p t I I}{ }_{c a t}{ }^{+}\right) / N$ for cells for cells carrying either pCON2 or pCON-S2. The proportion of cells carrying both plasmids was calculated as $P_{p 1 \& 2}=N_{n p t I I}{ }_{c a t}+/ N$. Plasmid loss was calculated as $P_{l o s s}=1-P_{p 1}-P_{p 2}-P_{p 1+2}$. Our evaluation regime included counting of preferably large and therefore reliable sample sizes for the determination of the different plasmid-carrying cell types. In cases when, due to variability in the plating, colony counts on double selective plates were slightly higher than on plates containing only one of the two antibiotics, counts were corrected toward the counting result on double selective medium in order to avoid negative numerical values in the calculation of the proportions.

\section{Serial Transfer Experiment/Short Term Evolution Experiment}

The evolution experiment was conducted under non-selective conditions and was founded with double resistant colonies from the preemptive plasmid fitness competition experiment between unstable pCON and stable pCON-S2. In three of the replicates of this experiment (i.e., replicates 4.1, 4.3, and 4.5) double resistant cells formed the vast majority in the analyzed colonies at the end of the competition. Because of this dominance, double resistant colonies could be readily picked from non-selective plates that were used to estimate the total number of cells within a colony at the end of the competition. For each the replicates, eight colonies were randomly picked and inoculated into $1 \mathrm{ml} \mathrm{LB}$ medium for overnight growth at $37^{\circ} \mathrm{C}$ with constant shaking. At the onset of the experiment samples were plated on double selective media to confirm the double resistant phenotype of the colonies. The overnight cultures were then serially propagated every $24 \mathrm{~h}$ in a volume of $1 \mathrm{ml} \mathrm{LB}$ medium for three additional transfers using a 1:1000 dilution factor. In total, we recorded $\sim 64$ generations ( $\sim 28$ generations for the first growth passage on solid medium and $\sim 9$ generations per transfer in liquid medium). Every day, the proportion of plasmid types in the populations was estimated from the proportion of host cells carrying either pCON or pCONS2 or both as described above for the plasmid competition experiments. At the end of the experiment an individual double resistant colony from four evolved populations of replicate 4.1 and replicate 4.3 were randomly chosen for plasmid isolation followed by diagnostic restriction enzyme digestion.

\section{Plasmid Stability Assay}

The plasmid loss frequency was estimated from the frequency of plasmid free cells occurring during overnight growth in non-selective media. To determine the loss frequency, cultures were inoculated from single colonies grown on selective media to ensure plasmid carriage. After $12 \mathrm{~h}$ growth in $37^{\circ} \mathrm{C}$ (approximately 8.5 generations), the cultures were serially diluted and plated on non-selective LB media. After overnight incubation, 100 colonies were picked and streaked on selective media (chloramphenicol $20 \mu \mathrm{g} / \mathrm{ml}$ ). Colonies grown on selective plates were counted as plasmid carrying. The loss frequency was calculated from plasmid-free cells (not resistant) and the total number of colonies tested.

\section{Fitness Experiments}

The relative fitness ( $w$ ) (Arjan et al., 1999) of the plasmidcarrying versus the ancestral plasmid-free strain (wt) was estimated by pairwise competition experiments. All competition experiments were initiated with a 1:1 mixture of 1:100 diluted plasmid-carrying strain and ancestral strain [E. coli MG1655 $\mathrm{Tm}^{r}$; (Wein et al., 2019)] from overnight cultures, in a total volume of $1 \mathrm{ml}$ of non-selective LB medium. The relative fitness of the plasmid host strains was calculated by evaluating cell counts at the time points 0 and $24 \mathrm{~h}$. The strains were distinguished through plating on nonselective (LB) and selective media (LB supplemented with trimethoprim $150 \mu \mathrm{g} / \mathrm{ml})$. The chromosomal integration $\left(\mathrm{Tm}^{r}\right)$ as well as the plasmid pCON have no measurable impact on the fitness of E. coli MG1655 [previously shown in (Wein et al., 2019)].

\section{Analysis of Plasmids by Restriction Enzyme Digestion}

Plasmids were extracted from stationary overnight cultures inoculated from single colonies and grown without selection using the GeneJET Plasmid Miniprep Kit (Thermo Fisher Scientific). Plasmid DNA was quantified using the Multiskan GO spectrophotometer instrument (Thermo Fisher Scientific). Plasmid conformations were analyzed by restriction enzyme digestions using PstI, ScaI or HindIII following the manufacturers' guidelines (New England Biolabs). Analytical digestions were electrophoresed against a known standard (Hyperladder ${ }^{\mathrm{TM}} 1 \mathrm{~Kb}$, Bioline) in $1 \times \mathrm{TBE}$ buffer at $4.3 \mathrm{~V} / \mathrm{cm}$ in $0.7 \%(\mathrm{w} / \mathrm{v})$ agarose gels containing $1 \mu \mathrm{g} / \mathrm{ml}$ ethidium bromide. Gels were destained with deionized water and documented using the ChemiDoc imaging system (Bio-Rad).

\section{Statistical Analysis}

Statistical tests and data analysis were performed in $\mathrm{R}$ version 3.5.3 ( $\mathrm{R}$ Core Team, 2020) and MatLab ${ }^{\odot}$ version R2015b.

\section{DATA AVAILABILITY STATEMENT}

All datasets generated for this study are included in the article/Supplementary Material. 


\section{AUTHOR CONTRIBUTIONS}

$\mathrm{NH}, \mathrm{TW}$, and TD designed the experiments. NH, TW, JE, and AG established and performed the experimental work and analyzed the data. TD supported the data analysis. All authors interpreted the results and wrote the manuscript.

\section{FUNDING}

This study was supported by the ZMB Young Scientist Grant 2018 (awarded to TW), the DFG focus program 1819 (Grant No. DA1202/2-1 awarded to TD), and the Leibniz ScienceCampus EvoLUNG (awarded to TD). AG is supported by the IMPRS for Evolutionary Biology.

\section{REFERENCES}

Antoine, R., and Locht, C. (1992). Isolation and molecular characterization of a novel broad-host-range plasmid from Bordetella bronchiseptica with sequence similarities to plasmids from gram-positive organisms. Mol. Microbiol. 6, 17851799. doi: 10.1111/j.1365-2958.1992.tb01351.x

Arjan, J. A., Visser, M., Zeyl, C. W., Gerrish, P. J., Blanchard, J. L., and Lenski, R. E. (1999). Diminishing returns from mutation supply rate in asexual populations. Science 283, 404-406. doi: 10.1126/science.283.5400.404

Baxter, J. C., and Funnell, B. E. (2014). Plasmid partition mechanisms. Microbiol. Spectr. 2, doi: 10.1128/microbiolspec.PLAS-0023-2014

Bedhomme, S., Perez Pantoja, D., and Bravo, I. G. (2017). Plasmid and clonal interference during post horizontal gene transfer evolution. Mol. Ecol. 26, 1832-1847. doi: 10.1111/mec.14056

Bergstrom, C. T., Lipsitch, M., and Levin, B. R. (2000). Natural selection, infectious transfer and the existence conditions for bacterial plasmids. Genetics 155, 1505-1519.

Bottery, M. J., Wood, A. J., and Brockhurst, M. A. (2017). Adaptive modulation of antibiotic resistance through intragenomic coevolution. Nat. Ecol. Evol. 1, 1364-1369. doi: 10.1038/s41559-017-0242-3

Buckner, M. M. C., Saw, H. T. H., Osagie, R. N., McNally, A., Ricci, V., Wand, M. E., et al. (2018). Clinically relevant plasmid-host interactions indicate that transcriptional and not genomic modifications ameliorate fitness costs of Klebsiella pneumoniae Carbapenemase-carrying plasmids. mBio 9:e02303-17. doi: 10.1128/mBio.02303-17

Carneiro, L. C., Mendes, P. V. C., Silva, S. P., Souza, G. R. L., and Bataus, L. A. M. (2016). Characterization of a cryptic and intriguing low molecular weight plasmid. Curr. Microbiol. 72, 351-356. doi: 10.1007/s00284-015-0959-7

Chaconas, G., and Norris, S. J. (2013). Peaceful coexistence amongst Borrelia plasmids: getting by with a little help from their friends? Plasmid 70, 161-167. doi: 10.1016/j.plasmid.2013.05.002

Chédin, F., Dervyn, R., Ehrlich, S. D., and Noirot, P. (1997). Apparent and real recombination frequencies in multicopy plasmids: the need for a novel approach in frequency determination. J. Bacteriol. 179, 754-761. doi: 10.1128/ jb.179.3.754-761.1997

Condit, R., and Levin, B. R. (1990). The evolution of plasmids carrying multiple resistance genes: the role of segregation, transposition, and homologous recombination. Am. Natural. 135, 573-596. doi: 10.1086/285063

Cooper, T. F., and Heinemann, J. A. (2000). Postsegregational killing does not increase plasmid stability but acts to mediate the exclusion of competing plasmids. Proc. Natl. Acad. Sci. U.S.A. 97, 12643-12648. doi: 10.1073/pnas. 220077897

Dionisio, F., Zilhão, R., and Gama, J. A. (2019). Interactions between plasmids and other mobile genetic elements affect their transmission and persistence. Plasmid 102, 29-36. doi: 10.1016/j.plasmid.2019.01.003

Doherty, M. J., Morrison, P. T., and Kolodner, R. (1983). Genetic recombination of bacterial plasmid DNA. Physical and genetic analysis of the products of

\section{ACKNOWLEDGMENTS}

We thank Fabian Nies for critical comments on the manuscript and Fenna T. Stücker for graphic illustrations. We thank Myriam Barz, Gabriela Rios Martinez, and Katrin Schumann for their assistance in the experimental work.

\section{SUPPLEMENTARY MATERIAL}

The Supplementary Material for this article can be found online at: https://www.frontiersin.org/articles/10.3389/fmicb. 2020.02062/full\#supplementary-material

plasmid recombination in Escherichia coli. J. Mol. Biol. 167, 539-560. doi: 10. 1016/s0022-2836(83)80097-7

Dower, W. J., Miller, J. F., and Ragsdale, C. W. (1988). High efficiency transformation of E. coli by high voltage electroporation. Nucleic Acids Res. 16, 6127-6145. doi: 10.1093/nar/16.13.6127

Flores, M., Mavingui, P., Perret, X., Broughton, W. J., Romero, D., Hernández, G., et al. (2000). Prediction, identification, and artificial selection of DNA rearrangements in Rhizobium: toward a natural genomic design. Proc. Natl. Acad. Sci. U.S.A. 97, 9138-9143. doi: 10.1073/pnas.97.16.9138

Fournes, F., Crozat, E., Pages, C., Tardin, C., Salomé, L., Cornet, F., et al. (2016). FtsK translocation permits discrimination between an endogenous and an imported Xer/dif recombination complex. Proc. Natl. Acad. Sci. U.S.A. 113, 7882-7887. doi: 10.1073/pnas.1523178113

Gerdes, K., Bech, F. W., Jørgensen, S. T., Løbner-Olesen, A., Rasmussen, P. B., Atlung, T., et al. (1986a). Mechanism of postsegregational killing by the hok gene product of the parB system of plasmid R1 and its homology with the relF gene product of the E. coli relB operon. Embo J. 5, 2023-2029. doi: 10.1002/j. 1460-2075.1986.tb04459.x

Gerdes, K., Rasmussen, P. B., and Molin, S. (1986b). Unique type of plasmid maintenance function: postsegregational killing of plasmid-free cells. Proc. Natl. Acad. Sci. U.S.A. 83, 3116-3120. doi: 10.1073/pnas.83.10.3116

Gitschlag, B. L., Kirby, C. S., Samuels, D. C., Gangula, R. D., Mallal, S. A., and Patel, M. R. (2016). Homeostatic responses regulate selfish mitochondrial genome dynamics in C. elegans. Cell Metab. 24, 91-103. doi: 10.1016/j.cmet.2016.06.008

González-Plaza, J. J., Blau, K., Milaković, M., Jurina, T., Smalla, K., and UdikovićKolić, N. (2019). Antibiotic-manufacturing sites are hot-spots for the release and spread of antibiotic resistance genes and mobile genetic elements in receiving aquatic environments. Environ. Int. 130, 104735. doi: 10.1016/j. envint.2019.04.007

Grote, J., Krysciak, D., Petersen, K., Güllert, S., Schmeisser, C., Förstner, K. U., et al. (2016). The absence of the $\mathrm{N}$-acyl-homoserine-lactone autoinducer synthase genes traI and ngrI increases the copy number of the cymbiotic plasmid in Sinorhizobium fredii NGR234. Front. Microbiol. 7:1858. doi: 10.3389/fmicb. 2016.01858

Gumpert, H., Kubicek-Sutherland, J. Z., Porse, A., Karami, N., Munck, C., Linkevicius, M., et al. (2017). Transfer and persistence of a multi-drug resistance plasmid in situ of the infant gut microbiota in the absence of antibiotic treatment. Front. Microbiol. 8:1852. doi: 10.3389/fmicb.2017.01852

Hall, J. P. J., Williams, D., Paterson, S., Harrison, E., and Brockhurst, M. A. (2017). Positive selection inhibits gene mobilisation and transfer in soil bacterial communities. Nat. Ecol. Evol. 1, 1348-1353. doi: 10.1038/s41559-017-0250-3

Hall, J. P. J., Wood, A. J., Harrison, E., and Brockhurst, M. A. (2016). Sourcesink plasmid transfer dynamics maintain gene mobility in soil bacterial communities. Proc. Natl. Acad. Sci. U.S.A. 113, 8260-8265. doi: 10.1073/pnas. 1600974113

Hanahan, D. (1983). Studies on transformation of Escherichia coli with plasmids. J. Mol. Biol. 166, 557-580. doi: 10.1016/s0022-2836(83)80284-8 
Heuer, H., Fox, R. E., and Top, E. M. (2007). Frequent conjugative transfer accelerates adaptation of a broad-host-range plasmid to an unfavorable Pseudomonas putida host. FEMS Microbiol. Ecol. 59, 738-748. doi: 10.1111/j. 1574-6941.2006.00223.x

Ilhan, J., Kupczok, A., Woehle, C., Wein, T., Hülter, N. F., Rosenstiel, P., et al. (2019). Segregational drift and the interplay between plasmid copy number and evolvability. Mol. Biol. Evol. 36, 472-486. doi: 10.1093/molbev/msy225

James, A. A., Morrison, P. T., and Kolodner, R. (1982). Genetic recombination of bacterial plasmid DNA. Analysis of the effect of recombination-deficient mutations on plasmid recombination. J. Mol. Biol. 160, 411-430. doi: 10.1016/ 0022-2836(82)90305-9

Jordt, H., Stalder, T., Kosterlitz, O., Ponciano, J. M., Top, E. M., and Kerr, B. (2020). Coevolution of host-plasmid pairs facilitates the emergence of novel multidrug resistance. Nat. Ecol. Evol. 364, 2275-2277. doi: 10.1038/s41559-020-1170-1

Jørgensen, T. S., Xu, Z., Hansen, M. A., Sørensen, S. J., and Hansen, L. H. (2014). Hundreds of circular novel plasmids and DNA elements identified in a rat cecum metamobilome. PLoS One 9:e87924. doi: 10.1371/journal.pone. 0087924

Kittell, B. L., and Helinski, D. R. (1991). Iteron inhibition of plasmid RK2 replication in vitro: evidence for intermolecular coupling of replication origins as a mechanism for RK2 replication control. Proc. Natl. Acad. Sci. U.S.A. 88, 1389-1393. doi: 10.1073/pnas.88.4.1389

Kolodner, R. (1980). Genetic recombination of bacterial plasmid DNA: electron microscopic analysis of in vitro intramolecular recombination. Proc. Natl. Acad. Sci. U.S.A. 77, 4847-4851. doi: 10.1073/pnas.77.8.4847

Krakauer, D. C., and Komarova, N. L. (2003). Levels of selection in positivestrand virus dynamics. J. Evol. Biol. 16, 64-73. doi: 10.1046/j.1420-9101.2003. 00481.x

Kusano, K., Naito, T., Handa, N., and Kobayashi, I. (1995). Restrictionmodification systems as genomic parasites in competition for specific sequences. Proc. Natl. Acad. Sci. U.S.A. 92, 11095-11099. doi: 10.1073/pnas.92. 24.11095

Loftie-Eaton, W., Bashford, K., Quinn, H., Dong, K., Millstein, J., Hunter, S., et al. (2017). Compensatory mutations improve general permissiveness to antibiotic resistance plasmids. Nat. Ecol. Evol. 1, 1354-1363. doi: 10.1038/s41559-0170243-2

Lopatkin, A. J., Huang, S., Smith, R. P., Srimani, J. K., Sysoeva, T. A., Bewick, S., et al. (2016). Antibiotics as a selective driver for conjugation dynamics. Nat. Microbiol. 1, 16044-16048. doi: 10.1038/nmicrobiol.2016.44

Lopatkin, A. J., Meredith, H. R., Srimani, J. K., Pfeiffer, C., Durrett, R., and You, L. (2017). Persistence and reversal of plasmid-mediated antibiotic resistance. Nat. Commun. 8:1689. doi: 10.1038/s41467-017-01532-1

Luria, S. E., and Delbrück, M. (1943). Mutations of bacteria from virus sensitivity to virus resistance. Genetics 28, 491-511.

Mahendra, C., Christie, K. A., Osuna, B. A., Pinilla-Redondo, R., Kleinstiver, B. P., and Bondy-Denomy, J. (2020). Broad-spectrum anti-CRISPR proteins facilitate horizontal gene transfer. Nat. Microbiol. 5, 620-629. doi: 10.1038/s41564-0200692-2

McKay, L. L., Baldwin, K. A., and Efstathiou, J. D. (1976). Transductional evidence for plasmid linkage of lactose metabolism in Streptococcus lactis C2. Appl. Environ. Microbiol. 32, 45-52. doi: 10.1128/aem.32.1.45-52.1976

Norberg, P., Bergström, M., Jethava, V., Dubhashi, D., and Hermansson, M. (2011). The IncP-1 plasmid backbone adapts to different host bacterial species and evolves through homologous recombination. Nat. Commun. 2:268. doi: 10 . $1038 /$ ncomms 1267

Paulsson, J. (2002). Multileveled selection on plasmid replication. Genetics 161, 1373-1384.

Pesesky, M. W., Tilley, R., and Beck, D. A. C. (2019). Mosaic plasmids are abundant and unevenly distributed across prokaryotic taxa. Plasmid 102, 10-18. doi: 10.1016/j.plasmid.2019.02.003

Pham, T. T. H., Rossi, P., Dinh, H. D. K., Pham, N. T. A., Tran, P. A., Ho, T. T. K. M., et al. (2018). Analysis of antibiotic multi-resistant bacteria and resistance genes in the effluent of an intensive shrimp farm (Long An, Vietnam). J. Environ. Manag. 214, 149-156. doi: 10.1016/j.jenvman.2018.02.089

Pinkney, M., Diaz, R., Lanka, E., and Thomas, C. M. (1988). Replication of mini RK2 plasmid in extracts of Escherichia coli requires plasmid-encoded protein TrfA and host-encoded proteins DnaA, B, G DNA gyrase and DNA polymerase III. J. Mol. Biol. 203, 927-938. doi: 10.1016/0022-2836(88) 90118-0

R Core Team (2020). R: A Language and Environment for Statistical Computing. Vienna: R Foundation for Statistical Computing.

Rodriguez-Beltran, J., Hernandez-Beltran, J. C. R., DelaFuente, J., Escudero, J. A., Fuentes-Hernandez, A., MacLean, R. C., et al. (2018). Multicopy plasmids allow bacteria to escape from fitness trade-offs during evolutionary innovation. Nat. Ecol. Evol. 2, 873-881. doi: 10.1038/s41559-018-0529-z

San Millan, A., Escudero, J. A., Gutierrez, B., Hidalgo, L., Garcia, N., Llagostera, M., et al. (2009). Multiresistance in Pasteurella multocida is mediated by coexistence of small plasmids. Antimicrob. Agents Chemother. 53, 3399-3404. doi: 10.1128/ AAC.01522-08

San Millan, A., Heilbron, K., and MacLean, R. C. (2014). Positive epistasis between co-infecting plasmids promotes plasmid survival in bacterial populations. ISME J. 8, 601-612. doi: 10.1038/ismej.2013.182

Silva, R. F., Mendonça, S. C. M., Carvalho, L. M., Reis, A. M., Gordo, I., Trindade, S., et al. (2011). Pervasive sign epistasis between conjugative plasmids and drug-resistance chromosomal mutations. PLoS Genet. 7:e1002181. doi: 10.1371/ journal.pgen.1002181

Sota, M., Yano, H., Hughes, J. M., Daughdrill, G. W., Abdo, Z., Forney, L. J., et al. (2010). Shifts in the host range of a promiscuous plasmid through parallel evolution of its replication initiation protein. ISME J. 4, 1568-1580. doi: 10. 1038/ismej.2010.72

Stark, W. M., Boocock, M. R., and Sherratt, D. J. (1992). Catalysis by site-specific recombinases. Trends Genet. 8, 432-439. doi: 10.1016/0168-9525(92)90327-z

Stevenson, C., Hall, J. P., Harrison, E., Wood, A., and Brockhurst, M. A. (2017). Gene mobility promotes the spread of resistance in bacterial populations. ISME J. 11, 1930-1932. doi: 10.1038/ismej.2017.42

Summers, D. K., Beton, C. W., and Withers, H. L. (1993). Multicopy plasmid instability: the dimer catastrophe hypothesis. Mol. Microbiol. 8, 1031-1038. doi: 10.1111/j.1365-2958.1993.tb01648.x

Taylor, D. R., Zeyl, C., and Cooke, E. (2002). Conflicting levels of selection in the accumulation of mitochondrial defects in Saccharomyces cerevisiae. Proc. Natl. Acad. Sci. U.S.A. 99, 3690-3694. doi: 10.1073/pnas.072660299

Wagner, E. G., Blomberg, P., and Nordström, K. (1992). Replication control in plasmid R1: duplex formation between the antisense RNA, CopA, and its target, CopT, is not required for inhibition of RepA synthesis. Embo J 11, 1195-1203. doi: 10.1002/j.1460-2075.1992.tb05160.x

Wang, Y. (2017). Spatial distribution of high copy number plasmids in bacteria. Plasmid 91, 2-8. doi: 10.1016/j.plasmid.2017.02.005

Wein, T., Hülter, N. F., Mizrahi, I., and Dagan, T. (2019). Emergence of plasmid stability under non-selective conditions maintains antibiotic resistance. Nat. Commun. 10:2595. doi: 10.1038/s41467-019-10600-7

Wein, T., Wang, Y., Hülter, N. F., Hammerschmidt, K., and Dagan, T. (2020). Antibiotics interfere with the evolution of plasmid stability. Curr. Biol. 30, doi: 10.1016/j.cub.2020.07.019

Yano, H., Wegrzyn, K., Loftie-Eaton, W., Johnson, J., Deckert, G. E., Rogers, L. M., et al. (2016). Evolved plasmid-host interactions reduce plasmid interference cost. Mol. Microbiol. 101, 743-756. doi: 10.1111/mmi.13407

Zar, J. H. (2013). Biostatistical Analysis, 5 Edn. Upper Saddle River: Pearson, 537-538.

Zhou, P., Zhai, Z., Yao, X., Ma, P., and Hao, Y. (2018). Characterization of a cryptic rolling-circle replication plasmid pMK8 from Enterococcus durans 1-8. Curr. Microbiol. 75, 1198-1205. doi: 10.1007/s00284-018-1509-x

Conflict of Interest: The authors declare that the research was conducted in the absence of any commercial or financial relationships that could be construed as a potential conflict of interest.

Copyright (c) 2020 Hülter, Wein, Effe, Garoña and Dagan. This is an open-access article distributed under the terms of the Creative Commons Attribution License (CC BY). The use, distribution or reproduction in other forums is permitted, provided the original author(s) and the copyright owner(s) are credited and that the original publication in this journal is cited, in accordance with accepted academic practice. No use, distribution or reproduction is permitted which does not comply with these terms. 